\title{
A New Antifungal Macrolide, Eushearilide, Isolated from Eupenicillium shearii
}

\author{
Tomoo Hosoe, Kazutaka Fukushima, Kayoko Takizawa, Takeshi Itabashi, \\ Nobuo Kawahara, Valerio Vidotto, Ken-ichi Kawai
}

Received: June 27, 2006 / Accepted: August 27, 2006

(C) Japan Antibiotics Research Association

\begin{abstract}
In screening for antifungal substances, a new macrolide, eushearilide (1), was isolated from Eupenicillium shearii IFM54447. The structure of $\mathbf{1}$ was established to be 24-membered macrolide having a non-conjugated diene and a choline phosphate ester moetiy on the basis of detailed investigation of NMR, UV, IR and MS spectral data. Compound $\mathbf{1}$ showed antifungal activity against various fungi and yeasts, including human pathogens Aspergillus fumigatus, Trichophyton spp. and Candida spp.
\end{abstract}

Keywords Eupenicillium shearii, Eushearilide, Macrolide, Antifungal activity

\section{Introduction}

The incidence of life-threatening fungal infections has steadily increased in immunocompromised hosts such as HIV infected persons and cancer and transplant patients [1]. Invasive pulmonary aspergillosis and Pneumocystis carinii pneumonia are leading causes of death in bone marrow transplant recipients and in HIV-infected patients, respectively. Moreover, resistance to the azoles, which are the most widely used antifungals today, is attracting much attention. Therefore, there is a continuing need for new antifungal agents to overcome these fungal diseases. Screening for new antifungal substances from fungal sources was carried out using pathogenic filamentous fungi, Aspergillus fumigatus Fresenius IFM41362 and Aspergillus niger Van Tieghem IFM41398, and/or pathogenic yeasts, Candida albicans (Robin) Berkhout ATCC90028 and Cryptococcus neoformans (Sanfelice) Vuillemin ATCC90112. The chloroform - methanol (1:1) extract of freshly isolated Eupenicillium shearii IFM54447, cultivated on rice for 21 days at $25^{\circ} \mathrm{C}$, showed antifungal activity against the above four test organisms. The purification of this extract led to the isolation of a new macrolide designated eushearilide (1) as the antifungal substance.

\section{Results and Discussion}

Eushearilide (1) was obtained as a white amorphous solid. High resolution time of flight mass spectrometry (HR-TOFMS) for 1 gave a quasimolecular ion $[\mathrm{M}+\mathrm{H}]^{+}$at $m / z$ 544.3757 (calcd 544.3762) corresponding to the molecular formula $\mathrm{C}_{29} \mathrm{H}_{54} \mathrm{NO}_{6} \mathrm{P}$, which was consistent with ${ }^{1} \mathrm{H},{ }^{13} \mathrm{C}$ and ${ }^{31} \mathrm{P}$ NMR spectra. Infrared (IR) absorption at 2920 and $2850 \mathrm{~cm}^{-1}$ suggested the presence of aliphatic moiety and that at $1730 \mathrm{~cm}^{-1}$ (strong) suggested the presence of an ester carbonyl. The ${ }^{1} \mathrm{H}$ NMR spectrum of $\mathbf{1}$ exhibited 54
T. Hosoe (Corresponding author), T. Itabashi, K. Kawai: Faculty of Pharmaceutical Sciences, Hoshi University, Ebara 2-4-41, Shinagawa-ku, Tokyo 142-8501, Japan,

E-mail: hosoe@hoshi.ac.jp

K. Fukushima, K. Takizawa: Research Center for Pathogenic Fungi and Microbial Toxicoses, Chiba University, Inohana 1-8-1, Chuo-ku, Chiba 260-8673, Japan
N. Kawahara: National Institute of Health Sciences, Kamiyoga 1-18-1, Setagaya-ku, Tokyo 158-8501, Japan

V. Vidotto: Dipartimento Discipline Medico-Chirurgiche, Sezione Malattie Infettive, Universita' deglistudi di Torino, Italia 
non-exchangeable protons, including three equivalent tertiary $(\delta 3.21)$ and a secondary $(\delta 1.19)$ methyl groups and four olefinic protons $(\delta 5.36,5.37,5.39$ and 5.50). The above olefins apparently possessed a Z-configuration at $\mathrm{C}$ -

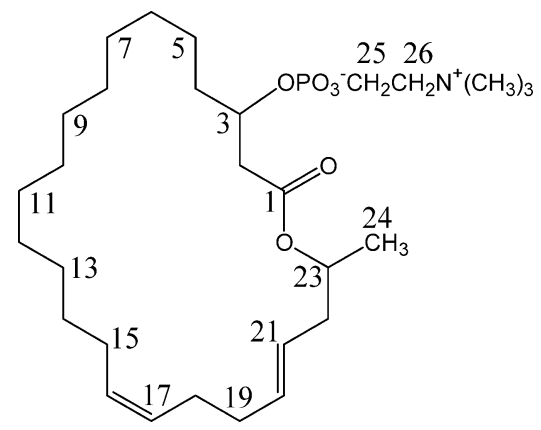

16, C-17 and an E-configuration at C-20, C-21 from the value of their coupling constants $\left(J_{16,17}=7.8 \mathrm{~Hz}\right.$ and $\left.J_{20,21}=15.1 \mathrm{~Hz}\right)$. The ${ }^{13} \mathrm{C}$ NMR spectrum of 1 showed four methyls ( $\delta 19.7$ and 54.7) including three equivalent methyls, 18 methylenes, two methines $(\delta 72.3$ and 74.1$)$ bearing oxygen functions, one carbonyl carbon $(\delta 171.8)$, and four tertiary olefinic carbon atoms $(\delta$ 126.5, 131.2, 131.8 and 134.7). A peak at $\delta-0.048 \mathrm{ppm}$ in the ${ }^{31} \mathrm{P}$ NMR spectrum of 1 showed the presence of a phosphoryl or phosphoric acid moiety.

From the analysis of the ${ }^{1} \mathrm{H}-{ }^{1} \mathrm{H}$ COSY and $\mathrm{HMBC}$ spectra (Fig. 2) of 1, the planar structure of eushearilide (1) was determined as a twenty-four membered macrolide with a non-conjugated diene and a choline phosphate ester moiety. The stereochemistry of $\mathbf{1}$ remains to be determined.

Fig. 1 Structure of eushearilide (1).

Table 1 Antifungal and antibacterial activities of eushearilide (1)

\begin{tabular}{|c|c|c|c|c|c|}
\hline Microorganisms & & $\begin{array}{l}\text { Inhibition zone } \\
\qquad(\mathrm{mm})\end{array}$ & Microorganisms & & $\begin{array}{l}\text { Inhibition zone } \\
\text { (mm) }\end{array}$ \\
\hline —Filamentous fungi- & & & & & \\
\hline$\langle$ Imperfect fungi and ascomycetes $\rangle$ & & & $\langle$ Zygomycetes $\rangle$ & & \\
\hline Alternaria alternata & IFM 41348 & 21 & Absidia corymbifera & IFM 41345 & 15 \\
\hline Arthroderma benhamiae & IFM 41160 & 20 & Cunninghamella elegans & IFM 47050 & 20 \\
\hline Aspergillus flavus & IFM 41935 & 24 & Mucor ramosissimus & IFM 46006 & 24 \\
\hline Aspergillus fumigatus & IFM 41362 & 14 & Rhizopus oryzae & IFM 40515 & 12 \\
\hline Aspergillus fumigatus & IFM 47078 & 15 & & & \\
\hline Aspergillus fumigatus & IFM 49896 & 21 & -Yeasts- & & \\
\hline Aspergillus fumigatus & IFM 51126 & 20 & Candida albicans & IFM 47945 & 8 \\
\hline Aspergillus fumigatus & IFM 51357 & 21 & Candida albicans & ATCC 90028 & 7 \\
\hline Aspergillus niger & IFM 41398 & 18 & Candida albicans & ATCC 90029 & 7 \\
\hline Aureobasidium pullulans & IFM 4802 & 20 & Candida dubliniensis & IFM 51756 & 11 \\
\hline Emericella nidulans & IFM 46997 & 12 & Candida glabrata & IFM 46888 & 7 \\
\hline Exophiala dermatitidis & IFM 41479 & 17 & Candida guilliermondii & IFM 46823 & (14) \\
\hline Fonsecaea pedrosoi & IFM 4887 & 11 & Candida kefyr & IFM 46921 & 11 \\
\hline Fusarium oxysporum f. sp. lactucae & IFM 53787 & 17 & Candida krusei & IFM 46834 & 7 \\
\hline Microsporum audouinii & IFM 41144 & 22 & Candida parapsilosis & IFM 46863 & 9 \\
\hline Microsporum canis & IFM 45108 & 23 & Candida tropicalis & IFM 46816 & 7.5 \\
\hline Penicillium citrinum & IFM 53298 & 19 & Cryptococcus neoformans & ATCC 90112 & 10.5 \\
\hline Penicillium islandicum & IFM 41098 & 11 & Cryptococcus neoformans & ATCC 90113 & 10 \\
\hline Penicilium marneffei & IFM 52703 & 20 & Saccharomyces cerevisiae & IFM 40210 & 7 \\
\hline Phialophora verrucosa & IFM 4928 & 11 & Trichosporon asahii var. asahii & IFM 48429 & (16) \\
\hline Pichia anomala & IFM 53788 & 9.5 & & & \\
\hline Pseudallescheria boydii & IFM 41901 & 26 & —Bacteria- & & \\
\hline Trichophyton mentagrophytes & IFM 40951 & 18 & Staphylococcus aureus & JCM 2151 & $(12)$ \\
\hline Trichophyton rubrum & IFM 45802 & 18 & Escherichia coli & JCM 1649 & - \\
\hline Trichophyton tonsurans & IFM 5275 & 20 & Pseudomonas aeruginosa & JCM 5962 & - \\
\hline Trichophyton verrucosum & IFM 46798 & 14 & & & \\
\hline
\end{tabular}

The parentheses mean hazy inhibition zone. 


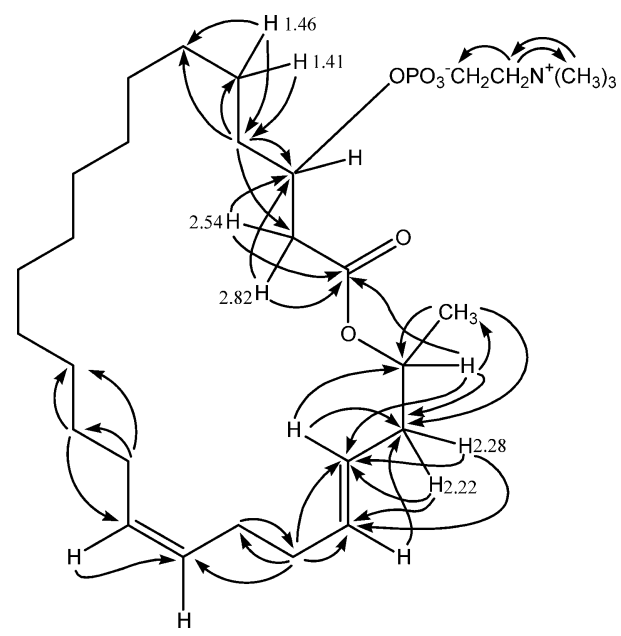

Fig. $2 \mathrm{HMBC}$ correlations in eushearilide (1).

\section{Antimicrobial Property}

Since eushearilide (1) was insoluble in water, the antimicrobial activity was determined by the paper disc method, as described in the previous paper $[2,3]$. The results are summarized in Table 1. Eushearilide (1) showed a broadrange of antifungal activity against various fungi and yeasts including human pathogens Aspergillus fumigatus, Trichophyton spp. and Candida spp. etc., whereas only a trace of the antibacterial activity was observed.

Although many macrolide antibiotics have several conjugated double bonds and amino sugar moieties in the molecular structure ( $c f$. amphotericin B [4]), eushearilide (1) is a macrolide antibiotic having a twenty-four membered ring, that has non-conjugated double bonds, no an amino sugar moiety and no hydroxyl groups on the ring structure. It is the first example to our knowledge of a twenty-four membered macrolide antibiotic having a choline phosphate ester moiety.

\section{Experimental}

ESI-TOF-MS was taken with a Bruker microTOF spectrometer. UV and IR spectra were recorded on a Hitachi U-3210 spectrometer and a JASCO IR-810 spectrometer, respectivity. ${ }^{1} \mathrm{H}$ and ${ }^{13} \mathrm{C}$ NMR spectra were recorded on a JEOL ECA-800 $\left({ }^{1} \mathrm{H}, 800.14 \mathrm{MHz}\right.$; ${ }^{13} \mathrm{C}, 201.20 \mathrm{MHz}$ ) spectrometer, using tetramethylsilane as an internal standard, and the ${ }^{31} \mathrm{P}$ NMR spectrum was recorded on a JEOL ECA-600 spectrometer. CD curves were determined on a JASCO J-600 spectropolarimeter. Column chromatography was performed using Wakogel C200 (Art. 237-00071, Wako). High performance liquid chromatography (HPLC) was performed with a Senshu
Scientific SSC-3160 pump (flow rate, $4 \mathrm{ml} / \mathrm{minute}$ ), equipped with a Shimamura YRD-883 RI detector. HPLC analytical condition of Eushearilide was as follows [column: Inertsil ODS-3, $4.6 \times 250 \mathrm{~mm}$, GL sciences Inc.; mobile phase: $\mathrm{MeOH}-\mathrm{H}_{2} \mathrm{O}(9: 1)$; flow rate: $1.0 \mathrm{ml} /$ minute; column oven temperature: $40^{\circ} \mathrm{C}$ ] TLC was conducted on pre-coated Kieselgel $60 \mathrm{~F}_{254}$ plates (Art. 5715; Merck) with solvent system $\mathrm{CHCl}_{3}-\mathrm{MeOH}-\mathrm{H}_{2} \mathrm{O}(6: 4: 1)$. Eushearilide was detected by spraying with $5 \% \mathrm{H}_{2} \mathrm{SO}_{4}$ and then heating.

\section{Isolation of Eushearilide (1) from $E$. shearii IFM54447}

E. shearii IFM54447, kept by The Research Center for Pathogenic Fungi and Microbial Toxicoses, Chiba University, was cultivated for 21 days at $25^{\circ} \mathrm{C}$ on rice (450 g, using 3 Roux flasks). The cultivated rice was extracted with $\mathrm{CHCl}_{3}-\mathrm{MeOH}(1: 1)$ and the evaporated extract suspended in water and partitioned with EtOAc. The EtOAc extract $(15 \mathrm{~g})$, which showed antifungal activity against $A$. fumigatus, was repeatedly chromatographed on silica gel (Wako, C-200) with $\mathrm{CHCl}_{3}-\mathrm{MeOH}$, followed by preparative reverse-phase HPLC [column: Senshu Pack pegasil-ODS, $10 \times 250 \mathrm{~mm}$; mobile phase: $\mathrm{MeOH}-\mathrm{H}_{2} \mathrm{O}$ $(9: 1)$ ] to give $\mathbf{1}(8 \mathrm{mg})$ along with a fraction including several lysophosphoglycerides.

Eushearilide (1): white amorphous solid; 1 was shown at Rt (15.2 minutes) and $\mathrm{Rf}$ value (0.42) in the above analytical condition. $[\alpha]_{\mathrm{D}}^{25}+12.8(c 0.75, \mathrm{MeOH})$; UV $(\mathrm{MeOH}): \lambda_{\max }(\log \varepsilon) 206$ (3.23), $225 \mathrm{~nm}$ (2.82); CD (MeOH): $\Delta \varepsilon(\mathrm{nm})+0.36$ (217); IR (film): $v_{\max } 3400$ (br), 2920 (s), 2850 (s), 1730 (s), 1230 (br), 1080 (s), 1060 (s), 850 (s) $\mathrm{cm}^{-1}$; HR-TOF-MS (ESI positive) $\mathrm{m} / z: 544.3757$ $[\mathrm{M}+\mathrm{H}]^{+}$; calcd. for $\mathrm{C}_{29} \mathrm{H}_{55} \mathrm{NO}_{6} \mathrm{P}: 544.3762$. ${ }^{1} \mathrm{H}-\mathrm{NMR}$ (800.14 MHz, CD $\mathrm{CD}_{3} \mathrm{OD}: \delta 1.19\left(3 \mathrm{H}, \mathrm{d}, J=6.4 \mathrm{~Hz}, 24-\mathrm{H}_{3}\right)$, $1.30\left(16 \mathrm{H}\right.$, br s, $6,7,8,9,10,11,12$, and $\left.13-\mathrm{H}_{2}\right), 1.36(2 \mathrm{H}$, $\left.\mathrm{m}, 14-\mathrm{H}_{2}\right), 1.41(1 \mathrm{H}, \mathrm{m}, 5-\mathrm{H}), 1.46(1 \mathrm{H}, \mathrm{m}, 5-\mathrm{H}), 1.64(2 \mathrm{H}$, $\left.\mathrm{m}, 4-\mathrm{H}_{2}\right), 2.00\left(2 \mathrm{H}\right.$, br dd, $\left.J=6.0,11.5 \mathrm{~Hz}, 15-\mathrm{H}_{2}\right), 2.06$ $\left(2 \mathrm{H}, \mathrm{m}, 18-\mathrm{H}_{2}\right), 2.07\left(2 \mathrm{H}, \mathrm{m}, 19-\mathrm{H}_{2}\right), 2.22(1 \mathrm{H}, \mathrm{ddd}, J=6.4$, 7.3, $14.0 \mathrm{~Hz}, 22-\mathrm{H}), 2.28(1 \mathrm{H}$, ddd, $J=6.9,7.3,14.0 \mathrm{~Hz}$, $22-\mathrm{H}), 2.54(1 \mathrm{H}, \mathrm{dd}, J=8.2,14.2 \mathrm{~Hz}, 2-\mathrm{H}), 2.82(1 \mathrm{H}, \mathrm{dd}$, $J=4.2,14.2 \mathrm{~Hz}, 2-\mathrm{H}), 3.21\left(9 \mathrm{H}, \mathrm{s}, 27-\mathrm{CH}_{3}\right), 3.62(2 \mathrm{H}, \mathrm{m}$, $\left.26-\mathrm{H}_{2}\right), 4.26\left(2 \mathrm{H}, \mathrm{m}, 25-\mathrm{H}_{2}\right), 4.54(1 \mathrm{H}, \mathrm{m}, 3-\mathrm{H}), 4.87$ $(1 \mathrm{H}$, br q, $J=6.4 \mathrm{~Hz}, 23-\mathrm{H}), 5.36(1 \mathrm{H}$, br d, $J=7.8 \mathrm{~Hz}$, $17-\mathrm{H}), 5.37(1 \mathrm{H}$, brd, $J=7.8 \mathrm{~Hz}, 16-\mathrm{H}), 5.39(1 \mathrm{H}, \mathrm{dt}$, $J=7.3,15.1 \mathrm{~Hz}, 21-\mathrm{H}), 5.50(1 \mathrm{H}, \mathrm{m}, 20-\mathrm{H}) \cdot ;{ }^{13} \mathrm{C}-\mathrm{NMR}$ (201.20 MHz, $\mathrm{CD}_{3} \mathrm{OD}$ ): $\delta 19.7$ (C-24), 25.4 (C-5), 28.5 (C13), 29.2, 29.4, 29.6, 29.7×2 and 29.8 (C-7 to C-12), 29.5 (C-14), 30.1 (C-6), 32.8 (C-15), 33.6 (C-18), 33.9 (C-19), 36.1 (C-4), 40.0 (C-22), 41.9 (C-2), 54.7 (C-27), 60.3 (C25), 67.5 (C-26), 72.3 (C-23), 74.1 (C-3), 126.5 (C-21), 131.2 (C-17), 131.8 (C-16), 134.7 (C-20), 171.8 (C-1). 


\section{Antibacterial and Antifungal Activities of 1}

Antibacterial and antifungal activities were qualitatively determined using the agar diffusion method with paper discs ( $6 \mathrm{~mm}$ in diameter), loaded with $40 \mu \mathrm{g}$ of 1 as described in the previous paper [2]. The test organisms used and the results are summarized in Table 1.

Acknowledgements We are grateful to Dr. H. Kasai and Miss N. Kobayashi of Hoshi University for NMR measurements. We also thank Prof. Y. Ebizuka of the University of Tokyo for mass measurements. This study was supported in part by Hoshi University Science/Technology Frontier Research Base and the Natural BioResouce Project-Pathogenic Microorganisms from the Ministry of Education, Science, Sports and Culture, Japan and by a Cooperative Research Pro\&shy;gram of Research Center for Pathogenic Fungi and Microbial Toxicoses, Chiba University (05-15).

\section{References}

1. Georgopapadakou NH, Walsh TJ. Antifungal agents: chemotherapeutic targets and immunologic strategies. J Antimicrob Agents Chemother 40: 279-291 (1996)

2. Hosoe T, Fukushima K, Itabashi T, Nozawa K, Takizawa K, Okada K, Takaki GMC, Kawai K. A new nonadride derivative, dihydroepiheveadride, as characteristic antifungal agent against filamentous fungi, isolated from unidentified fungus IFM52672. J Antibiot 57: 573-578 (2004)

3. Komai S, Hosoe T, Nozawa K, Okada K, Takaki GMC, Fukushima K, Miyaji M, Horie Y, Kawai K. Antifungal activity of pyranone and furanone derivatives, isolated from Aspergillus sp. IFM51759, against Aspergillus fumigatus. Mycotoxins 53: 11-18 (2003)

4. Inoue S, Sezaki M. Current antifungal antibiotics. Yukigoseikagakukyoukaishi 51: 327-349 (1993) 